\title{
Overview of ytterbium based transparent ceramics for diode pumped high energy solid-state lasers
}

\author{
Samuel Paul David, Venkatesan Jambunathan, Antonio Lucianetti, and Tomas Mocek \\ HiLASE Centre, Institute of Physics of the Czech Academy of Sciences, Za Radnicí 828/5, 25241, Dolní Břežany, Czech Republic \\ (Received 25 July 2018; revised 6 September 2018; accepted 9 November 2018)
}

\begin{abstract}
Development of high energy laser sources with nanosecond pulses at several hertz values for repetition rate has been very attractive in recent years due to their great potential for practical applications. With the recent advancement in fabricating large size laser quality transparent ceramics, diode pumped solid-state laser generating pulse energy of $100 \mathrm{~J}$ at $10 \mathrm{~Hz}$ has been recently realized at HiLASE center using Yb:YAG ceramic with Cr:YAG cladding. This review discusses Yb based high energy lasers, specific laser geometries for efficient thermal management and the role of transparent ceramics in such diode pumped high-energy-class solid-state lasers around the world.
\end{abstract}

Keywords: diode pumped solid-state lasers; high energy lasers; laser materials; transparent ceramics

\section{Introduction}

High power diode pumped solid-state lasers have gained more attention in recent years due to their potential scientific and technological applications ranging from laser fusion research to industrial materials processing. In addition, efficient, high energy, pulsed laser systems operating at high pulse repetition rates $(10 \mathrm{~Hz}$ and beyond) are the ideal pump sources for ultra-high intensity femtosecond petawattclass lasers generating high-brightness secondary radiation (X-ray, gamma-ray) and particle (electron, proton, ion, muon) sources. These have potential applications for novel medical therapies, and in high-resolution radiography and advanced imaging for industrial and security sectors. The improvements in semiconductor technology result in high power semiconductor laser diodes with high efficiency and power over long life times. High power laser diodes coupled by coupling optics or optical fiber are efficient pump sources for solid-state lasers based on materials such as crystals, glasses or ceramics. Compactness, portability and high efficiency make diode pumped solid-state lasers more desirable for wide range of applications. Such high energy compact lasers generating an average output power exceeding $100 \mathrm{~kW}$ with pulse energy of $500 \mathrm{~J}$ were chosen for military purposes as a high energy laser weaponry due to the ease of mounting them on moving vehicle or even on an aircraft ${ }^{[1]}$. Northrop Grumman demonstrated

Correspondence to: S. Paul David, HiLASE Centre, Institute of Physics of the Czech Academy of Sciences, Czech Republic.

Email: Samuel-Paul.David@hilase.cz
$105 \mathrm{~kW}$ output power by phase locking seven laser chain slabs (15 kW building blocks) pumped by high power laser diodes. In the field of plasma physics, laser-plasma interactions under high energy lasers can help scientists to understand relativistic plasma physics including compact particle accelerators and fast ignition of fusion targets. Extreme light infrastructure (ELI) will provide fs laser pulses with tens of joules of energy allowing generation of high energy density plasmas that are characterized by pressures above 1 Mbar $\left(1\right.$ bar $\left.=10^{5} \mathrm{~Pa}\right)$ or energy densities above $10^{11} \mathrm{~J} / \mathrm{m}^{3[2]}$. High-energy-class lasers with nanosecond pulses are in general limited to very low repetition rate in the order of few shots per minute or per hour, resulting in low average power. Such low average power lasers do not satisfy the requirements for advanced applications such as laser-driven inertial confinement fusion (ICF). Highly repetitive pulses $\geqslant 10 \mathrm{~Hz}$ with multi-joule energy are important for several applications ${ }^{[3]}$ such as laser induced damage threshold (LIDT) of optics ${ }^{[4]}$ and in aerospace industries to improve the fatigue life of metals and alloys by laser shock peening (LSP) technique ${ }^{[5]}$. With such wide application fields, several research groups around the world have been involved in prototyping high repetition high energy lasers. Bottlenecks to obtain high energy are laser gain media, pump sources and the cooling mechanism. A large size gain medium is necessary and such sizes can be easily made in glasses rather than crystalline form. The most common high energy laser based facilities such as National Ignition Facility (NIF) in USA, Shenguang-III in China, and Laser Mega Joule (LMJ) in France are based on Nd: glass ${ }^{[6]}$. 
However, these facilities can generate single shot every few hours to provide necessary time for cooling the poor thermal conductive glass media. Danson et al. gave an extensive overview of petawatt-class laser facilities around the world with energies ranging from $\mathrm{kJ}$ to $\mathrm{MJ}^{[7]}$. Compared to glasses, crystals have high thermal conductivity but are limited by their sizes because of the challenges in crystal growth and the growth striations present in the crystals. Crystals and glasses being the only choices for solid-state lasers, they have been extensively studied until the introduction of new class of solid-state gain media. In 1995, transparent ceramics joined as an alternative candidate to the realm of solid-state lasers along with the crystals and glasses ${ }^{[8]}$.

With continuous progress in transparent ceramic technology, lasers based on transparent ceramics have been popular over a couple of decades. This review article aims at bringing the recent progress in high energy lasers and the role of transparent ceramics to achieve high average power lasers. We also briefly discuss the impact of diode laser as a pump source and unique advantages of ytterbium ion with different hosts as active material. With a brief overview of several ongoing and upcoming high power laser projects globally, we have highlighted the recent demonstration of $1 \mathrm{~kW}$ average power from fully diode pumped solid-state lasers using $\mathrm{Yb}: \mathrm{Y}_{3} \mathrm{Al}_{5} \mathrm{O}_{12}$ (here after $\mathrm{Yb}: \mathrm{YAG}$ ) transparent ceramics at cryogenic temperature in HiLASE Center, Czech Republic.

\section{Diode as a pump source for solid-state lasers}

Semiconductor laser diodes generating a continuous wave (CW) output power greater than $500 \mathrm{~mW}$ are termed as high power diode lasers (HPDLs). Because of their reliability, compactness and portability, high power laser diodes are widely used as a pump source for solid-state and fiber lasers. In addition to that one can lock the wavelength in these diodes using volume Bragg grating (VBG). Such wavelength stabilization along with narrow spectral bandwidth $(<0.5 \mathrm{~nm})$ is very attractive for pumping solid-state lasers, especially to pump at specific wavelength such as the zero phonon line pumping of the gain medium ${ }^{[9]}$. Due to the improvements in semiconductor growth techniques such as metal organic chemical vapor deposition (MOCVD) and molecular beam epitaxy (MBE), semiconductor diode structures and laser cavity designs have been developed with variable atomic composition, doping concentration and precise atomic level layer thicknesses. Such refinements improve optical output power with significant electrical to optical power conversion efficiency (PCE) (>70\%). Such higher efficiency reduces the heat load and the material's internal losses. In a typical laser diode, optical emission takes place in one or more quantum wells of the $p-n$ junction. Among the several semiconductor layers, the quantum wells have the lowest energy gap and highest refractive index which helps to confine the photons within the quantum wells whose thickness can vary between few $\mathrm{nm}$ to few $\mu \mathrm{m}$. Output power from single emitter laser diode is improved by increasing the aperture/stripe width and by increasing the cavity length. Even though most practical laser diodes are available at near-IR (NIR) wavelength region between 800 and $980 \mathrm{~nm}$, new materials also extend the wavelength region covering visible (400-780 nm) and midinfrared region (1500-2300 $\mathrm{nm}$ ). These laser diodes operate both in classical $\mathrm{CW}$ and also in quasi-continuous wave (QCW) emission modes. In CW mode, the laser diode is driven continuously with an electrical current and thus the laser emits continuously. The efficiency and performance of laser diodes under $\mathrm{CW}$ mode are generally limited by the generation of heat. In QCW mode, diode driver current is applied for short time intervals which significantly reduces the thermal load compared to $\mathrm{CW}$ mode operation. Because of smaller duty cycle, heat can be efficiently removed which helps to reduce the thermal effects and to achieve a high peak power $^{[10]}$.

Continuous wave output power of more than $26 \mathrm{~W}$ with an output power facet density of $290 \mathrm{~mW} / \mu \mathrm{m}$ has been achieved at $940 \mathrm{~nm}$ using AlGaAs based broad-area single emitter laser diode with $90 \mu \mathrm{m}$ aperture size and a cavity length of $5 \mathrm{~mm}^{[11]}$. By combining several emitters, laser diode bars or diode stacks can generate higher power. A diode bar is a 1D array of several broad-area single emitters, whereas laser diode stack is a 2D array of several diode emitters arranged horizontally or vertically. Recently, at an injection current of $2 \mathrm{kA}$ an output power of $2 \mathrm{~kW}(200 \mu \mathrm{s}$, $10 \mathrm{~Hz}$ ) has been achieved using GaAs based $1 \mathrm{~cm}$ laser diode bar with double quantum-well design. A peak efficiency of $68 \%$ was achieved at an operating temperature of $203 \mathrm{~K}$ with the emission wavelength centered at $944 \mathrm{~nm}^{[12]}$. Multiple diode bars were used to generate multi $\mathrm{kW}$ laser power with high efficiency. JENOPTIK has demonstrated a pulse power of $4 \mathrm{~kW}$ using QCW diode stack containing eight QCW diode bars each generating $500 \mathrm{~W}(1 \mathrm{~ms}, 60 \mathrm{~Hz})$ pulse power at $940 \mathrm{~nm}$ which is suitable for pumping $\mathrm{Yb}$ doped high energy lasers. Wolz et al. from JENOPTIK demonstrated higher output power per chip with very less degradation ${ }^{[13]}$. Such high power and efficiency make them also attractive pump sources for high-energy-class laser facilities. Platz et al. built a fiber coupled diode pump module using modified emitter design as a pump source for Yb:YAG thin disc laser generating high average power. A peak power of $3.6 \mathrm{~kW}(1 \mathrm{~ms}, 100-200 \mathrm{~Hz})$ was delivered from a diode stack containing 28 emitters. Each emitter consists of InGaAs double quantum-well structure embedded in AlGaAs waveguide and delivers an output power of $130 \mathrm{~W}$ at $940 \mathrm{~nm}$ using an extreme double-asymmetric super large optical cavity $^{[14]}$. Morita et al. recently built a compact laser module capable of generating a peak power of $40 \mathrm{~kW}(1 \mathrm{~ms}, 40 \mathrm{~J})$ 
which will be used as a pump source for their upcoming $10 \mathrm{~J}$, $10 \mathrm{~Hz}$ laser system based on Yb:YAG ceramic based active mirror laser amplifier ${ }^{[15]}$.

Due to larger emitter width, higher fill factor and emitter count, HPDLs result in an elliptical output beam with a near diffraction-limit $\left(M^{2}<1.2\right)$ along the fast axis ( $y$-axis) and highly multimode $\left(M^{2}>800\right)$ along the slow axis $(x-$ axis). Even though a better beam quality can be achieved by reducing the emitter count and fill factor (the ratio of sum of emitter widths to the total width of the diode bar), this can also reduce the overall power. Beam quality can be significantly improved by employing an array of cylindrical microlenses along both the propagation axes. By placing fast-axis collimation lens to individual emitters, beams can be collimated along the fast axis. Once coupled into fiber, the beam quality and brightness can be maintained. Due to this reason, laser diodes have been found to be good alternative pump sources for solid-state laser to generate laser with high spectral and spatial characteristics compared to flash lamps. In addition to that, laser diodes have narrow band emission and require lower maintenance. The first diode pumped solid-state laser was demonstrated using $\mathrm{U}: \mathrm{CaF}_{2}$ at cryogenic liquid helium temperature ${ }^{[16]}$. The most common solid-state laser materials such as Nd:YAG and Yb:YAG can be efficiently pumped by laser diodes with emission spectra matching their absorption bands. Quantum-well laser diodes based on GaAs/AlGaAs are used for $\mathrm{Nd}$ based laser materials, whereas InGaAs/GaAs based laser diodes are used in $\mathrm{Yb}$ based materials because of the overlap between their absorption bands with the laser diode emission. The availability of high efficiency high power laser diodes leads to the implementation of successful and ongoing high energy laser projects such as POLARIS (Germany) ${ }^{[17]}$, LUCIA (France) $^{[18]}$, PEnELOPE (Germany) ${ }^{[19]}$ and HiLASE $(\text { Czechia })^{[20]}$. More details on such projects are discussed in the later sections.

\section{Ytterbium based transparent ceramics for solid-state laser materials}

Solid-state lasers producing high energy laser pulses at high repetition rates demand a large size gain media with extreme optical quality, high thermal conductivity and mechanical strength. Among several laser active ions, $\mathrm{Yb}^{3+}$ ion with two manifolds $\left({ }^{2} \mathrm{~F}_{7 / 2}\right.$ and $\left.{ }^{2} \mathrm{~F}_{5 / 2}\right)$ and energy separation of about $10,000 \mathrm{~cm}^{-1}$ is more desirable for high-energy-class lasers. Because of this simple electronic structure, it avoids unwanted thermal load due to nonlinear optical processes such as upconversion and excited state absorption (ESA), which are predominant with other common laser ions such as $\mathrm{Nd}^{3+}$. This also allows doping high concentration of $\mathrm{Yb}^{3+}$ ion without any concentration quenching. Additionally, a low quantum defect, longer life time $(\sim 1 \mathrm{~ms})$ and broadband emission lines of $\mathrm{Yb}^{3+}$ help to generate high energy laser pulses with relatively short durations ( $\sim$ ps). However, $\mathrm{Yb}^{3+}$ ion also suffers from few drawbacks due to its quasithree level nature of the ground state manifold (terminal laser level). At room temperature, the lower energy level gets thermally populated that causes reabsorption of laser wavelength reducing the population inversion and eventually increasing the laser threshold. The best way to avoid such thermal population is to operate the laser at cryogenic temperature. At cryogenic temperature, the $\mathrm{Yb}$ doped gain materials act as a four level laser medium, which reduces the reabsorption of laser wavelength. In addition, cryogenic cooling helps in improving the spectroscopic and thermooptic properties of the laser gain medium. All these effects result in overall system laser performance. Fan et al. studied several $\mathrm{Yb}$ doped materials at cryogenic temperature and mentioned the possibility of increasing the average power by more than 100 times at such cryogenic operation ${ }^{[21]}$. With the availability of high power laser diodes that match the absorption band of $\mathrm{Yb}^{3+}$, solid-state lasers based on $\mathrm{Yb}^{3+}$ find more attraction due to high energy storage and high wall plug efficiency.

Solid-state lasers have been built with $\mathrm{Yb}^{3+}$ ion doped in glasses, single crystals and transparent ceramics. Yb doped glasses such phosphates, tellurites, fluorides and silicate glasses have been extensively studied for laser development because of their cost effectiveness and broad emission spectrum which is ideal for ultrashort lasers ${ }^{[22-24]}$. Glasses require significant cooling for high average power lasers due to their poor thermal conductivity. Similarly, $\mathrm{Yb}$ ions have been doped in several crystalline materials for their potential laser applications. Using quasi-three level laser model, Brenier et al. evaluated several $\mathrm{Yb}$ doped single crystals based on their output power and slope efficiency. According to their analysis, double tungstate crystals (KGW and $\mathrm{KYW})$ and sesquioxides $\left(\mathrm{Y}_{2} \mathrm{O}_{3}, \mathrm{Sc}_{2} \mathrm{O}_{3}, \mathrm{Lu}_{2} \mathrm{O}_{3}\right)$ showed the highest laser potentialities. These materials can be grown as high quality single crystals, however growing large size single crystals will be demanding due to the technical difficulties and huge $\cos ^{[25]}$. Over the years, YAG gained more attention because of its broad absorption band, a high emission cross section, higher thermal conductivity and the ability to grow such crystals in large dimensions. Yb:YAG broad absorption at $940 \mathrm{~nm}$ matches well with emission of high power laser diodes based on InGaAs. Lacovara et al. demonstrated the first room temperature diode pumped $\mathrm{Yb}$ :YAG laser with an efficiency of $31 \%$ pumped by a single InGaAs laser diode ${ }^{[26]}$. Longer life time along with smaller quantum defect and mechanical robustness enables Yb:YAG to generate higher energy compared to Nd:YAG. Low quantum defect reduces the heat generation by four times compared to $\mathrm{Nd}: \mathrm{YAG}^{[27]}$.

Just above a couple of decades ago, Japanese scientists introduced the laser quality transparent ceramics into the solidstate laser domain which were earlier based only on single 
crystals and glasses. With controlled processing steps right from powder preparation followed by pressing, sintering and polishing, cubic materials without any secondary phase and porosity can be made transparent. For the fabrication of the earliest transparent YAG ceramics, two Japanese groups adapted different powder synthesis techniques to form YAG powders keeping the subsequent processing steps such as forming, pressing and sintering common. Ikesue et al. from World Lab Co., Ltd fabricated YAG transparent ceramics by hot pressing the mixture of precursors such as $\mathrm{Y}_{2} \mathrm{O}_{3}, \mathrm{Al}_{2} \mathrm{O}_{3}$ and $\mathrm{Nd}_{2} \mathrm{O}_{3}{ }^{[8]}$, whereas Yanagitani and Yagi from Konoshima Chemicals developed ceramics using YAG powders synthesized by co-precipitation technique. Ceramics were made at a temperature much lower than its melting point, at a shorter fabrication time and in desirable shapes. Among several oxide ceramics developed over the years, yttrium aluminum garnet and yttrium oxide ceramics are considered the best laser host materials followed by other sesquioxides such as scandia and lutetia ${ }^{[28]}$. Even though sesquioxides show better thermal properties than YAG, fabrication of large aperture sesquioxides ceramics still remains a challenge. Yttrium aluminum garnet ceramics have gained its spot in all the major high power lasers based on transparent ceramics.

Takaichi et al. demonstrated the first CW laser using 1\% Yb:YAG (1.6 mm thick) ceramic under $940 \mathrm{~nm}$ fiber coupled laser diode pumping with a slope efficiency of $26 \%$ and a maximum power of $345 \mathrm{~mW}^{[29]}$. Yb:YAG ceramics also show good optical and mechanical properties as single crystals. These ceramics can be exceptionally made in large apertures and also with high dopant concentration which are important requirements for building high power lasers. High quality Yb:YAG ceramics with high $\mathrm{Yb}$ concentration (up to 20 at.\%) were developed by the same group and they demonstrated diode pumped dual-wavelength microchip laser operation with a maximum slope efficiency of $79 \%$ using 9.8 at.\% doped $1 \mathrm{~mm}$ thick Yb:YAG ceramic at room temperature ${ }^{[30]}$. An efficiency of $52 \%$ was achieved with $20 \%$ Yb doped YAG ceramics under laser diode pumping and the ceramics have been found to perform better than the crystalline counterpart ${ }^{[31]}$. The effect of dopant concentration on the spectroscopic and thermal properties of YAG was reviewed extensively by Ref. [32]. Composite all-ceramic Yb:YAG rod with doped core surrounded by undoped cladding was used to generate output powers of $520 \mathrm{~W}$ (peak power) in quasi-CW mode at an input power of $997 \mathrm{~W}$ (peak power) with $56 \%$ efficiency ${ }^{[33]}$. The ceramic composite used in microchip configuration and the output power against the incident power are shown in Figures 1(a) and 1(b).

Over the years, Yb:YAG ceramics gained more attention in the laser community focused on high power generation. Yb:YAG ceramic rod was proposed in their conceptual design of GENBU laser in Japan aiming to generate $1 \mathrm{~kJ}$ laser pulses at a repetition rate of $100 \mathrm{~Hz}$ which are desirable for fusion energy reactor driver ${ }^{[34]}$. These lasers are diode pumped and the ceramics are operated in active mirror configuration. Recently, an average power of $1 \mathrm{~kW}$ has been achieved in HiLASE in diode pumped laser configuration using large aperture Yb:YAG ceramics. Specific details are discussed in the latter part of this article.

Other than YAG ceramics, ytterbium has also been explored in other host materials such as sesquioxides in transparent ceramic form. $\mathrm{Yb}: \mathrm{Y}_{2} \mathrm{O}_{3}$ ceramic 20 at. $\%$ was successfully developed and a successful laser oscillation with an efficiency of $32 \%$ was demonstrated even before Yb:YAG ceramic laser ${ }^{[35]}$. This was followed by $\mathrm{Yb}: \mathrm{Lu}_{2} \mathrm{O}_{3}$ ceramic laser with an efficiency of $53 \%{ }^{[36]}$. Apart from better thermal conductivity, $\mathrm{Yb}$ doped sesquioxides have large value of nonlinear refractive index that can enhance Kerrlens and phase modulation effects. Such nonlinear effects along with better thermal conductivity make sesquioxides suitable for the development of ultrashort lasers with high average power. Tokurakawa et al. extensively studied on the generation of sub-100 fs lasers using $\mathrm{Yb}$ doped sesquioxides under laser diode pumping ${ }^{[37]}$. Laser pulses with pulse duration as short as 53 fs were achieved in diode pumped (a)

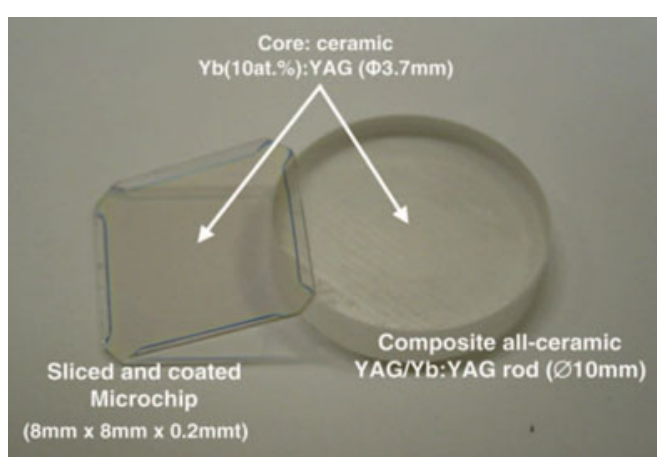

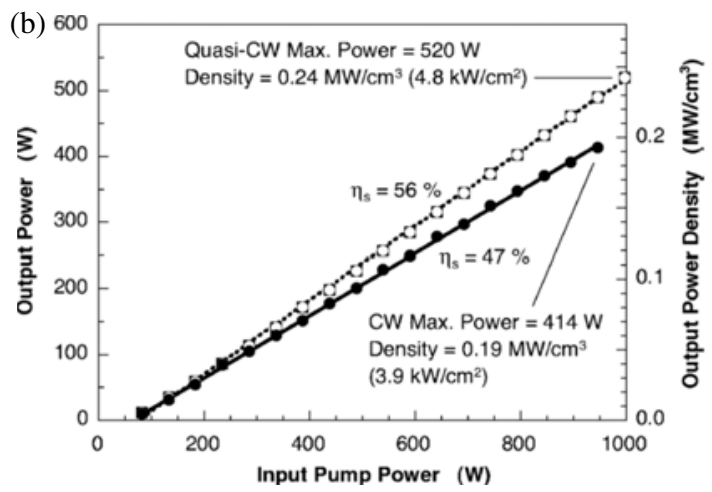

Figure 1. (a) Composite ceramic gain media used to achieve an output power of $520 \mathrm{~W}$ in microchip laser. (b) An output power of $520 \mathrm{~W}$ using YAG/Yb:YAG composite gain media. Reprinted with permission from Ref. [33]. 
mode locked operation of $\mathrm{Yb}: \mathrm{Sc}_{2} \mathrm{O}_{3}$ ceramics $^{[38]}$. Other than cubic oxides, ytterbium has been an attractive laser ion for non-cubic transparent ceramics as well. Non-cubic apatite ceramics such as SFAP and FAP doped with $\mathrm{Yb}$ were fabricated by electric field and magnetic field assisted sintering, respectively ${ }^{[39,40]}$. Kilowatt peak power laser has been recently demonstrated by using orientation controlled non-cubic FAP ceramics by Sato et al. Such non-cubic $\mathrm{Yb}$ :FAP ceramics were fabricated by slip-casting the slurry under intense magnetic field followed by pre-sintering in air and hot isostatic pressing in argon atmosphere. In addition to oxide transparent ceramics, $\mathrm{Yb}$ doped fluoride ceramic lasers are also gaining attention for high energy and ultrashort lasers. Recently, an efficiency of $69.5 \%$ was achieved using $\mathrm{Yb}$ doped $\mathrm{CaF}_{2}-\mathrm{LaF}_{3}$ transparent ceramic under laser diode pumping ${ }^{[41]}$.

\section{High energy solid-state lasers}

As mentioned in the introduction part, high efficiency nanosecond multi-joule class lasers with high repetition rates $(>10 \mathrm{~Hz})$ are becoming more and more attractive in the laser community compared to single-shot laser facilities. Diode pumped solid-state lasers generating nanosecond pulses with energy in tens of joules and more are highly demanding in a wide variety of application fields. The major challenges in achieving such high energy at several hertz high repetition rate are LIDT of the optics and gain media, thermal effects of the laser material due to the pumping process and the amplified spontaneous emission (ASE). Using a careful amplifier design including the size and shape of the gain medium, LIDT and ASE can be controlled. For instance, laser induced damage of optics can be avoided by increasing the pump spot size, whereas ASE can be suppressed using an edge-cladding absorbing layer around the gain media. Intensive works have been carried out to suppress thermal effects which result in the degradation of beam quality due to thermal distortions such as thermally induced birefringence ${ }^{[42]}$. Severe thermal gradients along the gain media can also result in thermally induced crack of the material, especially for the laser operating at high average power. Various laser amplifier configurations such as laser rod, zig-zag slab, active mirror slab, and face cooled multi-slab amplifiers have been investigated for efficient heat removal for such high average power lasers. In this section, we focus on the two most common amplifier configurations in practice for diode pumped high energy solid-state lasers: active mirror slab amplifier and multi-slab amplifier.

When introduced by General Electric Co. in the late 1960s, active mirror laser amplifiers did not have much success due to the dielectric coating issues on the gain media. Within few years, significant progress has been made on demonstration of high average power laser using active mirror amplifier (AMA). In AMA systems, the active mirror slab or disk has high aspect ratio with thickness in the order of $\mathrm{mm}$ to $\mathrm{cm}$ and a diameter of few centimeters. The pump beam is directed on the rear side of the slab which is coated with dichroic coatings having high transmission for the pump wavelength and high reflectivity for the laser beam. The front face of the slab is coated with maximum reflection for pump wavelength and a high transmission for the laser beam. AMA is a double-pass design that enables efficient energy extraction from the gain medium with twice power gain during each pass. Classically AMA laser was performed by flashlamp pumping with uniform transverse thermal distribution across the gain media and was cooled by direct contact of the coolant along the rear surface of the slab ${ }^{[43]}$. A smooth thermal gradient reduces the thermal lensing effect. Brown et al. improved the laser configuration and cooling system and they demonstrated an average power of $120 \mathrm{~W}$ using LHG-5 glass pumped by Xe flashlamp in sandwich configuration using two laser disks pumped in a back-to-back fashion ${ }^{[44]}$. Vetrovec introduced an elementary scaling theory which states the maximum available average power depends linearly on the pump density and disk volume that is dependent both on disc thickness and diameter ${ }^{[45]}$.

Diode pumped AMA systems generate high efficiency and excellent beam quality compared to flashlamp pumped systems. Nd:glass has been a common gain medium for AMA system but it suffers from poor thermal conductivity and low emission cross section that limit in achieving $\mathrm{kW}$ of average power. Crystalline gain media such as Nd:YAG and Yb:YAG also have been explored in AMA laser for high repetition rate high energy pulse generation but the power scaling is generally limited by the aperture dimension. Generation of nanosecond laser pulses with energy of $100 \mathrm{~J}$ requires a gain medium with an aperture size of more than $5 \mathrm{~cm}$ to survive the LIDT value of $\sim 3 \mathrm{~J} / \mathrm{cm}^{2}{ }^{[46]}$. Transparent ceramics offer unique advantages not only to make large aperture gain media but also have the potential to achieve composite gain media. Therefore, a combination of diode pump and transparent ceramics is attractive for high average power lasers in active mirror concept and its improved versions such as total-reflection active mirror or thin disc lasers. LUCIA laser is one of the most popular diode pumped solid-state lasers aiming to deliver high energy nanosecond laser pulses at a repetition rate of $10 \mathrm{~Hz}$. It is also a part of the HiPER project which is dedicated to developing laser-driven inertial fusion energy sources ${ }^{[47]}$. LUCIA laser consists of a cavity dumped master oscillator power amplifier (MOPA) followed by two pre-amplifier stages and a main amplifier stage. Pumped by $10 \mathrm{~W}$ laser diode, MOPA generates $0.5 \mathrm{~nJ}$ laser pulses at $10 \mathrm{~Hz}$ repetition rate using a $10 \% \mathrm{Yb}$ :YAG single crystal disk $(3.5 \mathrm{~mm}$ thick $\times 25 \mathrm{~mm}$ diameter), whereas pre-amplifier stages use two $2 \% \mathrm{Yb}$ :YAG single crystal disks $(3 \mathrm{~mm}$ thick $\times 30 \mathrm{~mm}$ diameter). After multiple passes in the pre-amplifier stages, 
(a)

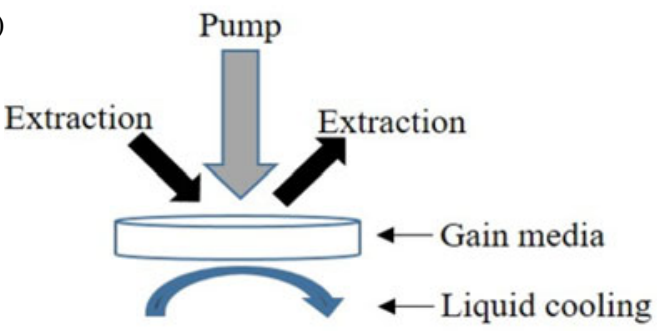

(b)

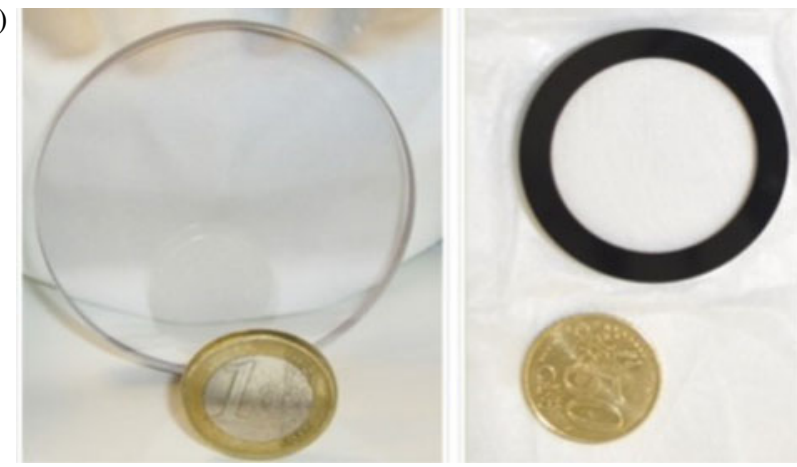

Figure 2. (a) Schematic of AMA concept used for LUCIA laser. (b) Crystalline and ceramic composites used in LUCIA laser to deliver an energy of $14 \mathrm{~J}$. Reprinted with permission from Ref. [18].

laser pulse reaches $590 \mathrm{~mJ}$ of energy and enters the main power amplifier stage which is pumped by laser diode with an average power of $600 \mathrm{~W}$. To avoid parasitic lasing (PL) due to ASE under such high power pumping, large diameter $2 \%$ Yb:YAG crystal $(7 \mathrm{~mm}$ thick $\times 60 \mathrm{~mm}$ diameter $)$ was considered. Due to the difficulties in growing such a large diameter single crystal, an alternative solution using $\mathrm{Cr}^{4+} / \mathrm{Yb}^{3+}$ :YAG composite ceramics ( $45 \mathrm{~mm}$ diameter) was found to generate same amount of laser energy. An energy of $14 \mathrm{~J}$ with more than $20 \%$ efficiency has been demonstrated using such composite ceramics. Schematic AMA concept used in LUCIA and the composite crystalline and ceramic media used to demonstrate an energy of $14 \mathrm{~J}$ are shown in Figures 2(a) and 2(b) ${ }^{[17]}$.

Compared to this first generation composite ceramics with $\mathrm{Cr}^{4+}$ layer $(0.25$ at.\%) having a radial thickness of $5 \mathrm{~mm}$, second generation transparent ceramics with $20 \mathrm{~mm}$ radial thickness of $\mathrm{Cr}^{4+}$ layer $(<0.1$ at.\%) shows higher gain $^{[48]}$. An increase in thickness and a decrease in doping level of $\mathrm{Cr}^{4+}$ layer reduce the thermal concentration and thermally induced stress along the $\mathrm{Yb}^{3+} / \mathrm{Cr}^{4+}$ interface. Along with improvement in cooling system, LUCIA laser aims at generating $100 \mathrm{~J} / \mathrm{ns}$ laser pulses at $10 \mathrm{~Hz}$.

Another laser geometry which provides efficient heat removal from the gain media and is ideal for high average power laser generation is multi-slab laser geometry. A slab laser allows effective cooling on both the faces by making direct contact with the coolant. Conventionally, a laser slab is cut at Brewster angle and the laser beam passes in zig-zag fashion within the slab by total internal reflection. In the first end pumped zig-zag slab laser, an average power of $150 \mathrm{~W}$ was achieved by Goodno et al. using a composite YAG/Yb:YAG crystalline slab ${ }^{[49]}$. Zig-zag geometry suffers from poor pump efficiency. Innovative laser slab (Innoslab) concept was used to improve heat removal by conductive cooling along both the slab faces and partial end pumping by a laser diode stack. Du et al. achieved an efficiency of $48 \%$ using Nd:YAG crystalline slab in their first ever demonstration using this concept ${ }^{[50]}$. A face pumped and face cooled laser has less thermal induced optical distortion and is suitable for high average power laser. Combined with efficient thermal management, power scaling can be done by increasing the number of slabs. Lawrence Livermore National Laboratory (LLNL), USA adopted this multi-slab concept in their diode pumped solid-state laser system named Mercury. They demonstrated an average power above $600 \mathrm{~W}(61 \mathrm{~J} @ 10 \mathrm{~Hz})$ using fourteen gascooled Yb:S-FAP crystalline slabs distributed along two amplifier stages of the system. The amplifier allowed the beam to undergo four multi-passes through the gain medium.

Similar projects such as PEnELOPE and POLARIS from Germany are fully diode pumped laser systems aiming at generating very high peak power in the order of terawatt. They adopted chirped pulse amplification (CPA) to amplify fs laser pulses by gain media based on glasses. PEnELOPE ${ }^{[19]}$ is a direct diode pumped CPA laser system based on active mirror/multi-slab architecture to generate laser pulses of energy $150 \mathrm{~J}$ at $1 \mathrm{~Hz}$ repetition rate with a pulse duration of $120 \mathrm{fs}$. Gain media, including Yb doped glasses such as phosphate and fluoride phosphate and crystalline $\mathrm{CaF}_{2}$ were preferred because of their relatively lower refractive indices which enable an easy index matching and a better limiting aspect ratio. $5 \mathrm{~mm}$ thick $\mathrm{Yb}: \mathrm{CaF}_{2}$ crystals of $5.5 \mathrm{~cm}$ and $11 \mathrm{~cm}$ with broad-band gain were chosen for two different multi-pass amplifier stages. The slabs are gas-cooled longitudinally to reduce thermally induced effects such as depolarization losses, stress induced birefringence and thermally induced stress ${ }^{[19]}$. Similarly, POLARIS project at Helmholtz-Institute Jena aims to achieve a peak power of $250 \mathrm{TW}$ using gain media based on $\mathrm{Yb}$ doped fluoride phosphate glass and $\mathrm{CaF}_{2}$ single crystals. Pumped by $940 \mathrm{~nm}$ high power laser diode stacks, POLARIS currently generates laser pulses of energy $16.7 \mathrm{~J}$ with a pulse duration of $98 \mathrm{fs}$ which corresponds to a peak power of $170 \mathrm{TW}^{[17]}$.

The scalability of this multi-slab concept has been adopted by another high energy DPSSL called Diode Pumped Optical Laser for Experiments (DiPOLE) using transparent ceramics of Yb:YAG. Figure 3 shows a schematic multislab amplifier design adopted for DiPOLE laser. Thick gain medium in the design provides high pump absorption and narrow gaps between the slabs enable the flow of high pressure He gas for efficient heat removal from the slab face. An end pumped system experiences severe heat load in 
the front face that causes longitudinal temperature gradients along the gain media and results in severe degradation in thermo-optical quality of the material. In order to maintain uniform thermal load, laser architecture can be considered in different ways: by using composite ceramic gain media with a varied distribution of dopant ions along the pumping direction; or by using tape cast multilayered ceramics with different layers having different dopant concentrations ${ }^{[51]}$; or by using multi-slab laser media having slabs of different concentrations. By these techniques, it is possible to build laser architecture to generate high power laser beam with good beam quality without degrading the thermal properties of the laser material. The DiPOLE project in Central Laser Facility (CLF) aimed at building high average power laser using the latter technique, i.e., by using cryogenically cooled multi-slab Yb:YAG ceramic ${ }^{[52]}$. Figure 3 shows multi-slab amplifier design used for DiPOLE project. Other major issues to be controlled are the undesirable ASE and PL that reduce the average energy storage density of the excited laser level. Especially for high energy lasers using large aperture amplifier and high pumping density, large beam aperture increases the amplification path length of photons emitted spontaneously that leads to severe ASE losses and should be curbed completely for efficient laser operation ${ }^{[53]}$. Among several approaches such as edge-face tilting or using anti-ASE cap ${ }^{[54]}$, ASE absorber cladding layer around the core laser rods in the transverse direction to pumping has been found to be effective in suppressing such ASE. For instance, Sm:YAG has been found to be a suitable cladding layer for Nd:YAG core laser rod compared to $\mathrm{Co}^{3+}$ and $\mathrm{Cr}^{4+}$ based cladding layers ${ }^{[55]}$. Cladding Sm:YAG layer has dual advantages: a strong absorption at lasing wavelength of Nd:YAG, i.e., at $1064 \mathrm{~nm}$ and a high transmission at the pump diode wavelength, i.e., at $808 \mathrm{~nm}^{[56]}$. These advantages help to reduce thermal loading and limit the wave-front distortion which is critical in high power solidstate lasers. High average power of $67 \mathrm{~kW}$ based on Nd:YAG ceramic slabs with Sm:YAG edge cladding has been achieved in solid-state heat capacity laser in LLNL ${ }^{[57]}$.

Similarly, $\mathrm{Cr}^{4+}$ :YAG having strong absorption at $1030 \mathrm{~nm}$ was considered as the cladding layer for Yb:YAG. It was found in $\mathrm{Cr}^{4+}$ and $\mathrm{Yb}$ co-doped YAG crystal amplifier, that depletion of energy due to ASE could be significantly reduced with an optimum $\mathrm{Cr}^{4+}$ concentration which is dependent on the aperture size and $\mathrm{Yb}$ concentration in core laser rod ${ }^{[53]}$. The main drawback of such co-doped materials is strong absorption of $\mathrm{Cr}^{4+}$ at $940 \mathrm{~nm}$, the pump wavelength for $\mathrm{Yb}$ :YAG and affects the performance of laser. Magdalena et al. have studied numerically the role of $\mathrm{Cr}^{4+}$ :YAG cladding layer on ASE around $\mathrm{Yb}: \mathrm{YAG}$ ceramic $\operatorname{slab}^{[58,59]}$. It has been found that ASE leads to a decrease in population inversion $\left(N_{2}\right)$ in the center part of the rectangular ceramic slabs. Under such ASE, change in population

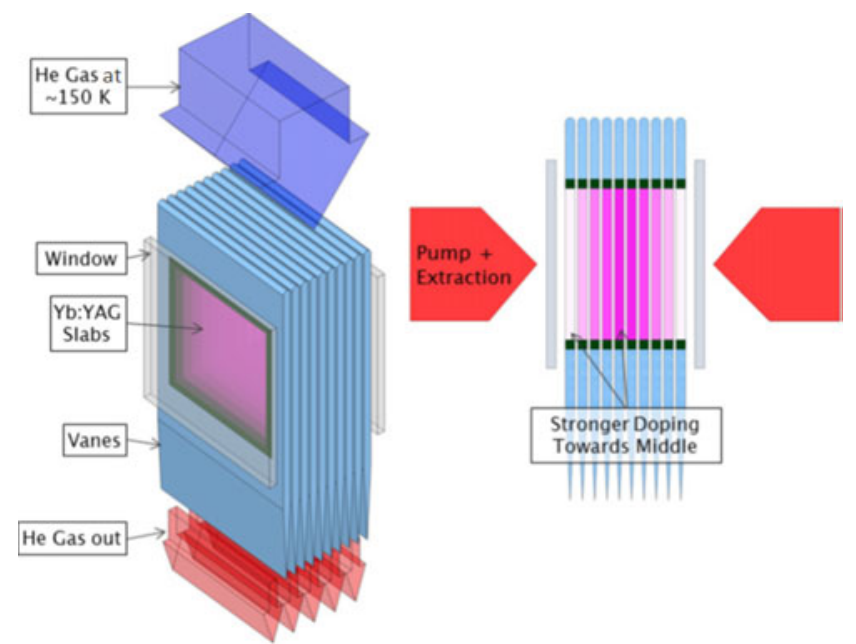

Figure 3. Multi-slab amplifier design adopted for DiPOLE laser systems. Reprinted with permission from Ref. [52].

inversion is given by

$$
\frac{\mathrm{d} N_{2}}{\mathrm{~d} t}=-\frac{N_{2}}{\tau}-\frac{\sigma_{e a} l_{a} N_{2}^{2}}{\tau}+R_{p}
$$

where $\tau$ is the upper laser level life time, $\sigma_{e a}$ is the average emission cross section, $l_{a}$ is the average path length of photons inside the gain medium and $R_{P}$ is the pumping rate per volume. The second term in the above equation represents ASE losses in the medium. According to their calculations, $0.3 \% \mathrm{Cr}^{4+}$ :YAG (3 cm thick) layer could absorb $99 \%$ of photons before they return to Yb:YAG region. Similarly Ertel et al. from STFC, UK using numerical models explored several aspects of amplifier architecture starting from optimization of pump efficiency such as pump duration, pump bandwidth, pump intensity, and doping concentration of gain media, their dimensions and the laser operating temperature ${ }^{[60]}$. Based on their models, they succeeded in achieving $10.8 \mathrm{~J} / 10 \mathrm{~ns}$ laser pulses at $10 \mathrm{~Hz}$ with $22.5 \%$ optical to optical conversion efficiency using five Yb:YAG ceramic discs ( $45 \mathrm{~mm}$ diameter $\times 5 \mathrm{~mm}$ thick) with $5 \mathrm{~mm}$ wide $0.25 \% \mathrm{Cr}^{4+}$ :YAG as the ASE absorber cladding layer as shown in Figure 4. Cr has a strong absorption at $1030 \mathrm{~nm}$ with an absorption coefficient of $6 \mathrm{~cm}^{-1}$. These $55 \mathrm{~mm}$ diameter ceramic discs are the amplifying media in the main amplifier, whereas the booster amplifier preceding this is based on $2.5 \% \mathrm{Yb}$ :YAG crystal. In order to maintain uniform pump absorption and heat load distribution along the longitudinal pumping direction, inner two discs are highly doped with $\mathrm{Yb}^{3+}(2$ at.\%), whereas the outer discs are 1.1 at. $\% \mathrm{Yb}: \mathrm{YAG}^{[61]}$. The amplifier was pumped on two sides by two $940 \mathrm{~nm}$ pump diodes delivering $20 \mathrm{~kW}$ power each and the laser was operated at $140 \mathrm{~K}$ by high pressure streaming of $\mathrm{He}$ gas through the disc gaps of $1.5 \mathrm{~mm}$ to provide distributed face cooling. With this demonstration 


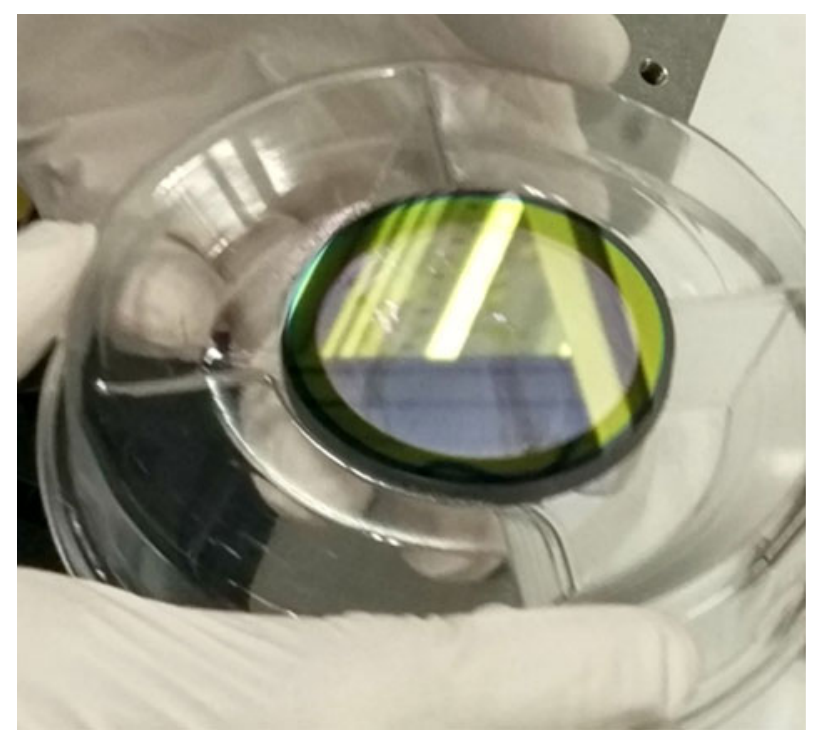

Figure 4. One of the ceramic discs used in DiPOLE-10 to generate an energy of $10 \mathrm{~J}$ at $10 \mathrm{~Hz}$.

and a stable laser operation for more than $50 \mathrm{~h}$, STFC and HiLASE took a major step to scale the laser energy to $100 \mathrm{~J}$ at $10 \mathrm{~Hz}$ using the same multi-slab architecture based on Yb:YAG transparent ceramics.

\section{Kilowatt class diode pumped solid-state laser in Hi- LASE using transparent ceramics}

$100 \mathrm{~J}$ HiLASE laser (also called DiPOLE100) consists of three components: $100 \mathrm{~mJ} / 10 \mathrm{~ns}$ seed laser pulses, preamplifier stage delivering $10 \mathrm{~J}$ and finally cryogenic main amplifier delivering $100 \mathrm{~J} / 10 \mathrm{~ns}$ laser pulses. The first component consists of seed source, regenerative pre-amplifier based on $\mathrm{Yb}: \mathrm{CaF}_{2}$ and multi-pass booster amplifier based on Yb:YAG crystal. More details on this first component can be found elsewhere ${ }^{[62]}$. The $10 \mathrm{~J}$ amplifier is based on gas-cooled multi-slab laser system using Yb:YAG ceramic circular discs as mentioned above. Amplification is achieved by seven passes through the amplifier which increases energy extraction with less ASE losses. With further optimization, an efficiency of $27 \%$ was achieved for this $10 \mathrm{~J}$ pre-amplifier. The beam is sent for further amplification to $100 \mathrm{~J}$ in cryogenic main amplifier which is a scaled up version of $10 \mathrm{~J}$ pre-amplifier. Unlike circular ceramic discs in $10 \mathrm{~J}$ amplifier head, rectangular ceramic slabs are used for $100 \mathrm{~J}$ amplifier. It consists of $8.5 \mathrm{~mm}$ thick six rectangular Yb:YAG ceramic slabs with $\mathrm{Cr}^{4+}$ :YAG cladding layer. Yb doped core region in the slab is $100 \mathrm{~mm}$ wide, whereas cladding layer is $10 \mathrm{~mm}$ wide. Figure 5 shows ceramic slabs used in $100 \mathrm{~J}$ main amplifier.

The slabs are similarly cooled by passing high pressure $\mathrm{He}$ gas through the $2 \mathrm{~mm}$ slab gaps and the laser amplification is

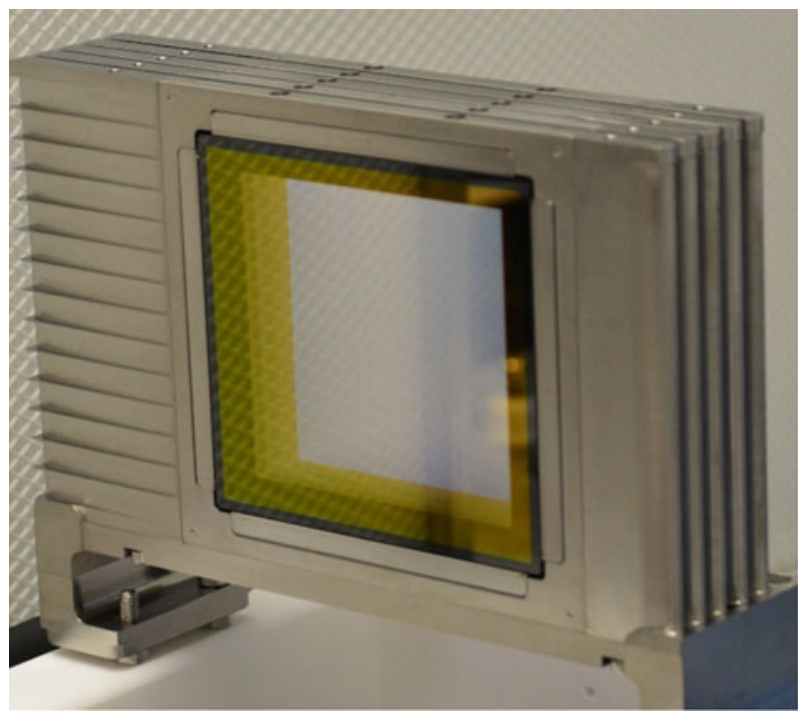

Figure 5. Yb:YAG ceramic slabs used to achieve $100 \mathrm{~J}$ average energy per pulse in HiLASE.

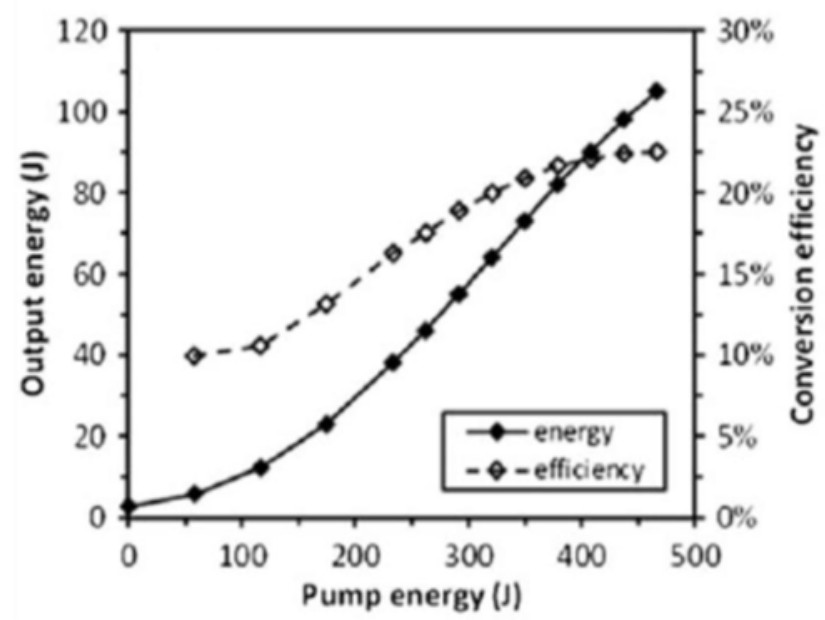

Figure 6. Output energy of $100 \mathrm{~J}$ against the pump energy. Reprinted with permission from Ref. [20].

done at $150 \mathrm{~K}$. The slabs are once again doped with different $\mathrm{Yb}$ concentrations in order to achieve uniform longitudinal absorption and heat load. Two center slabs are $1 \% \mathrm{Yb}$ doped followed by $0.6 \%$ and $0.4 \% \mathrm{Yb}$ doped slabs, whereas $\mathrm{Cr}^{4+}$ concentration for the cladding layer is chosen to provide an absorption coefficient of $\sim 3 \mathrm{~cm}^{-1}$ at $1030 \mathrm{~nm}^{[63]}$. Laser pulses with $105 \mathrm{~J}$ output energy at $10 \mathrm{~Hz}$ were achieved at an incident pump energy of $465 \mathrm{~J}$ with an optical to optical conversion efficiency of $22.5 \%$. An average power of exceeding $1 \mathrm{~kW}$ was demonstrated for the first time in diode pumped solid-state laser scheme ${ }^{[20]}$. Figure 6 shows the energy and efficiency from the main cryogenic amplifier. Both ceramic discs and rectangular ceramic slabs for the respective $10 \mathrm{~J}$ and $100 \mathrm{~J}$ amplifier heads were supplied by Konoshima Chemicals, Japan. The stable laser operation exceeding $1 \mathrm{~h}$ with such high efficiency is attractive for several scientific, medical and industrial applications. 


\section{Conclusions}

Applications ranging from industrial to high-field research demand lasers operating at high average power. Diode pumped solid-state lasers are the potential contender for the development of efficient and compact laser systems for such applications. Demand for large aperture gain media and an efficient way of heat removal have been the limiting factors for generating high repetition pulses with energies ranging from tens of joules to $\mathrm{kJ}$. With the advancement in ceramic technology, it is possible to engineer the laser gain media not only in terms of size but also in terms of composition and combination of layers such as composite ceramics. The crucial roles of ytterbium based materials, high power laser diodes and transparent ceramic gain media toward the recent progress in high energy lasers have been discussed. We also discussed two laser geometries in practice for high average power (HAP) lasers: active mirror and multi-slab amplifier concepts. Finally, recent achievement of kilowatt class laser operation in HiLASE using multi-slab large aperture Yb:YAG transparent ceramic clearly demonstrates a great potential for scaling this laser to the multi-100 J/10 Hz level.

\section{Acknowledgements}

This work was co-financed by the European Regional Development Fund and the state budget of the Czech Republic (project HiLASE CoE: Grant No. CZ.02.1.01/0.0/ 0.0/15_006/0000674) and by the European Union's Horizon 2020 research and innovation programme under grant agreement No. 739573. This work was also supported by the Ministry of Education, Youth and Sports of the Czech Republic (Programmes NPU I Project No. LO1602, and Large Research Infrastructure Project No. LM2015086).

\section{References}

1. J. Hecht, Opt. Photonics News 14, 42 (2003).

2. S. Weber, S. Bechet, S. Borneis, L. Brabec, M. Bučka, E. Chacon-Golcher, M. Ciappina, M. DeMarco, A. Fajstavr, K. Falk, E.-R. Garcia, J. Grosz, Y.-J. Gu, J.-C. Hernandez, M. Holec, P. Janečka, M. Jantač, M. Jirka, H. Kadlecova, D. Khikhlukha, O. Klimo, G. Korn, D. Kramer, D. Kumar, T. Lastovička, P. Lutoslawski, L. Morejon, V. Olšovcová, M. Rajdl, O. Renner, B. Rus, S. Singh, M. Šmid, M. Sokol, R. Versaci, R. Vrána, M. Vranic, J. Vyskočil, A. Wolf, and Q. Yu, Matter Radiat. Extremes 2, 149 (2017).

3. Y. Gao, Y. Wang, A. Chan, M. Dawson, and B. Greene, Laser Phys. Lett. 14, 035803 (2017).

4. J. Vanda, J. Sevčík, E. Pupka, M. Sčiuka, A. Melninkaitis, M. Divoky, V. Jambunathan, S. Bonora, V. Śkoda, A. Lucianetti, D. Rostohar, T. Mocek, and V. Sirutkaitis, High Power Laser Sci. Eng. 4, e11 (2016).

5. H. Kamkarrad, S. Narayanswamy, and M. Keshmiri, J. Laser Micro Nanoeng. 10, 291 (2015).
6. V. Samarkin, A. Alexandrov, G. Borsoni, T. Jitsuno, P. Romanov, A. Rukosuev, and A. Kudryashov, High Power Laser Sci. Eng. 4, e4 (2016).

7. C. Danson, D. Hillier, N. Hopps, and D. Neely, High Power Laser Sci. Eng. 3, e3 (2015).

8. A. Ikesue, T. Kinoshita, K. Kamata, and K. Yoshida, J. Am. Ceram. Soc. 78, 1033 (1995).

9. V. Jambunathan, L. Horackova, P. Navratil, A. Lucianetti, and T. Mocek, IEEE Photonics Technol. Lett. 28, 1328 (2016).

10. M. Zorn, Laser Technik J. 12, 25 (2015).

11. I. B. Petrescu-Prahova, P. Modak, E. Goutain, D. Silan, D. Bambrick, J. Riordan, T. Moritz, S. D. McDougall, B. Qiu, and J. H. Marsh, Proc. SPIE 7198, 71981I (2009).

12. C. Frevert, F. Bugge, S. Knigge, A. Ginolas, G. Erbert, and P. Crump, Proc. SPIE 9733, 97330L (2016).

13. M. Wolz, A. Pietrzak, A. Kindsvater, J. Wolf, J. Meusel, R. Hulsewede, and J. Sebastian, Proc. SPIE 9513, 95130E (2015).

14. R. Platz, B. Eppich, J. Rieprich, W. Pittroff, G. Erbert, and P. Crump, High Power Laser Sci. Eng. 4, e3 (2016).

15. T. Morita, T. Sekine, Y. Takeuchi, Y. Hatano, T. Kurita, Y. Tamaoki, Y. Mizuta, Y. Kabeya, M. Kurata, K. Kawai, Y. Muramatsu, T. Iguchi, K. Iyama, Y. Zheng, and Y. Kato, in Laser Congress 2017 (ASSL, LAC) (OSA, 2017), paper JTu2A.31.

16. R. J. Keyes and T. M. Quist, Appl. Phys. Lett. 4, 50 (1964).

17. M. Hornung, H. Liebetrau, S. Keppler, A. Kessler, M. Hellwing, F. Schorcht, G. A. Becker, M. Reuter, J. Polz, J. Korner, J. Hein, and M. C. Kaluza, Opt. Lett. 41, 5413 (2016).

18. T. Gonçalvès-Novo, D. Albach, B. Vincent, M. Arzakantsyan, and J.-C. Chanteloup, Opt. Express 21, 855 (2013).

19. M. Siebold, F. Roeser, M. Loeser, D. Albach, and U. Schramm, Proc. SPIE 8780, 878005 (2013).

20. P. Mason, M. Divoky, K. Ertel, J. Pilar, T. Butcher, M. Hanus, S. Banerjee, J. Phillips, J. Smith, M. De Vido, A. Lucianetti, C. Hernandez-Gomez, C. Edwards, T. Mocek, and J. Collier, Optica 4, 438 (2017).

21. T. Y. Fan, D. J. Ripin, R. L. Aggarwal, J. R. Ochoa, B. Chann, M. Tilleman, and J. Spitzberg, IEEE J. Sel. Top. Quantum. Electron. 13, 448 (2007).

22. X. Zou and H. Toratani, Phys. Rev. B 52, 15889 (1995).

23. P. Nandi and G. Jose, IEEE J. Quantum Electron. 42, 1115 (2006).

24. X. E. Su, Y. X. Zhou, M. H. Zhou, Y. R. Zhu, P. Cheng, Z. Z. Zhou, and N. J. Wang, J. Alloys Compd. 739, 149 (2018).

25. A. Brenier and G. Boulon, J. Alloys Compd. 323, 210 (2001).

26. P. Lacovara, H. K. Choi, C. A. Wang, R. L. Aggarwal, and T. Y. Fan, Opt. Lett. 16, 1089 (1991).

27. T. Y. Fan, IEEE J. Quantum Elecron. 29, 1457 (1993).

28. A. Goldstein and and A. Krell, J. Am. Ceram. Soc. 99, 3173 (2016)

29. K. Takaichi, H. Yagi, J. Lu, A. Shirakawa, K. Ueda, T. Yanagitani, and A. A. Kaminskii, Phys. Status Solidi A 200, R5 (2003).

30. J. Dong, A. Shirakawa, K. Ueda, H. Yagi, T. Yanagitani, and A. A. Kaminskii, Appl. Phys. Lett. 89, 091114 (2006).

31. J. Dong, A. Shirakawa, K. I. Ueda, H. Yagi, T. Yanagitani, and A. A. Kaminskii, Opt. Lett. 32, 1890 (2007).

32. T. Taira, IEEE J. Quantum Elect. 13, 798 (2007).

33. M. Tsunekane and T. Taira, Appl. Phys. Lett. 90, 121101 (2007).

34. J. Kawanaka, R. Yasuhara, Y. Takeuchi, S. J. Pearce, T. Kawashima, and H. Kan, in European Conference on Lasers and Electro-Optics and the European Quantum Electronics Conference (IEEE, 2009), p. 1. 
35. J. R. Lu, K. Takaichi, T. Uematsu, A. Shirakawa, M. Musha, K. Ueda, H. Yagi, T. Yanagitani, and A. A. Kaminskii, Japan J. Appl. Phys. 41, L1373 (2002).

36. K. Takaichi, H. Yagi, A. Shirakawa, K. Ueda, S. Hosokawa, T. Yanagitani, and A. A. Kaminskii, Phys. Status Solidi A 202, R1 (2005).

37. M. Tokurakawa, A. Shirakawa, K. Ueda, H. Yagi, S. Hosokawa, T. Yanagitani, and A. A. Kaminskii, Opt. Lett. 33, 1380 (2008)

38. M. Tokurakawa, A. Shirakawa, K. Ueda, H. Yagi, M. Noriyuki, T. Yanagitani, and A. A. Kaminskii, Opt. Express 17, 3353 (2009)

39. Y. Q. Wu, Opt. Mater. Express 4, 2026 (2014).

40. Y. Sato, M. Arzakantsyan, J. Akiyama, and T. Taira, Opt. Mater. Express 4, 2006 (2014).

41. S. Kitajima, K. Yamakado, A. Shirakawa, K. Ueda, Y. Ezura, and H. Ishizawa, Opt. Lett. 42, 1724 (2017).

42. O. Slezak, A. Lucianetti, and T. Mocek, IEEE J. Quantum Electron. 50, 1 (2014).

43. J. A. Abate, L. Lund, D. Brown, S. Jacobs, S. Refermat, J. Kelly, M. Gavin, J. Waldbillig, and O. Lewis, Appl. Opt. 20, 351 (1981).

44. D. C. Brown, R. Bowman, J. Kuper, Kotik K. Lee, and J. Menders, Appl. Opt. 25, 612 (1986).

45. J. Vetrovec, Proc. SPIE 4270, 45 (2001).

46. D. Albach and J.-C. Chanteloup, Opt. Express 23, 570 (2015).

47. J.-C. Chanteloup, D. Albach, A. Lucianetti, K. Ertel, S. Banerjee, P. D. Mason, C. Hernandez-Gomez, J. L. Collier, J. Hein, and M. Wolf, J. Phys. Conf. Ser. 244, 012010 (2010).

48. S. Marrazzo, T. Gonçalvès-Novo, F. Millet, and J.-C. Chanteloup, Opt. Express 24, 12651 (2016).

49. G. D. Goodno, S. Palese, J. Harkenrider, and H. Injeyan, Opt. Lett. 26, 1672 (2001)

50. K. Du, N. Wu, J. Xu, J. Giesekus, P. Loosen, and R. Poprawe, Opt. Lett. 23, 370 (1998)

51. A. Ikesue and Y. L. Aung, Nat. Photonics 2, 721 (2008).
52. K. Ertel, S. Banerjee, P. D. Mason, P. J. Phillips, C. Hernandez-Gomez, and J. L. Collier, in Conference on Lasers and Electro-Optics Pacific Rim (IEEE, 2013), paper TuD1-2.

53. J. G. Zheng, X. W. Yan, X. Y. Jiang, Z. G. Wang, W. T. Duan, and M. Z. Li, Proc. SPIE 8080, 80800S (2011).

54. D. Kouznetsov and J. F. Bisson, J. Opt. Soc. Am. B 25, 338 (2008).

55. H. Yagi, J. F. Bisson, K. Ueda, and T. Yanagitani, J. Lumin. 121, 88 (2006).

56. R. Huss, R. Wilhelm, C. Kolleck, J. Neumann, and D. Kracht, Opt. Express 18, 13094 (2010).

57. R. M. Yamamoto, B. S. Bhachu, K. P. Cutter, S. N. Fochs, S. A. Letts, C. W. Parks, M. D. Rotter, and T. F. Soules, in Advanced Solid-State Photonics (OSA, 2008), paper WC5.

58. M. Sawicka, M. Divoky, J. Novak, A. Lucianetti, B. Rus, and T. Mocek, J. Opt. Soc. Am. B 29, 1270 (2012).

59. A. Lucianetti, M. Sawicka, O. Slezak, M. Divoky, J. Pilar, V. Jambunathan, S. Bonora, R. Antipenkov, and T. Mocek, High Power Laser Sci. Eng. 2, e13 (2014).

60. K. Ertel, S. Banerjee, P. D. Mason, P. J. Phillips, M. Siebold, C. Hernandez-Gomez, and J. C. Collier, Opt. Express 19, 26610 (2011).

61. S. Banerjee, K. Ertel, P. D. Mason, P. J. Phillips, M. De Vido, J. M. Smith, T. J. Butcher, C. Hernandez-Gomez, R. J. S. Greenhalgh, and J. L. Collier, Opt. Express 23, 19542 (2015).

62. P. D. Mason, S. Banerjee, K. Ertel, P. J. Phillips, T. J. Butcher, J. M. Smith, M. De Vido, S. Tomlinson, O. Chekhlov, W. Shaikh, S. Blake, P. Holligan, M. Divoky, J. Pilar, C. Hernandez-Gomez, R. J. S. Greenhalgh, and J. L. Collier, Proc. SPIE 9513, 951302 (2015).

63. S. Banerjee, P. D. Mason, K. Ertel, P. J. Phillips, M. De Vido, O. Chekhlov, M. Divoky, J. Pilar, J. Smith, T. Butcher, A. Lintern, S. Tomlinson, W. Shaikh, C. Hooker, A. Lucianetti, C. Hernandez-Gomez, T. Mocek, C. Edwards, and J. L. Collier, Opt. Lett. 41, 2089 (2016). 